\title{
Vital fluorescent dyes for study of silicifying organisms
}

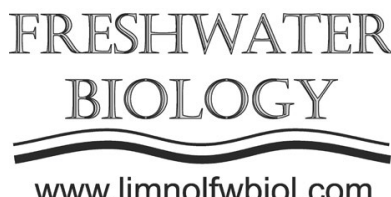

\author{
Annenkov V.V.*๑, Zelinskiy S.N. ${ }^{\oplus}$, Pal’shin V.A. ${ }^{\oplus}$, Avezova T.N., Danilovtseva E.N. ${ }^{\oplus}$
}

Limnological Institute, Siberian Branch of the Russian Academy of Sciences, Ulan-Batorskaya Str., 3, Irkutsk, 664033, Russia

\begin{abstract}
The mini-review describes application of vital fluorescent dyes to study silica biosynthesis in diatom algae and siliceous sponges. Mechanisms of these processes are not clear and study of growing siliceous constructions in living organisms is complicated with silica transparency and presence of mature elements (siliceous valves of diatoms and spicules of sponges). Specific fluorescence dyes stain growing siliceous elements of skeletons and also primary siliceous particles which are formed during silicon capture from the environment. Vital fluorescent dyes based on 7-nitro-2,1,3-benzoxadiazole, pyridyl oxazole and coumarin fluorophores are described as well as examples of application of these dyes to study diatom algae and siliceous sponges.
\end{abstract}

Keywords: fluorescent dyes, diatom algae, siliceous sponges, biosilicification

\section{Introduction}

A lot of living organisms use siliceous constructions for building of important elements of their bodies. The most known are diatom algae ( $>20 \%$ of primary oxygen and organic carbon production (Treguer et al., 1995), siliceous sponges (water filterers and a source of biological active compounds), rise and horsetail. For many decades scientists try to answer question: how these organisms build highly ordered constructions from silica at ambient temperatures? This question is intriguing and important because:

- biosilica shows a great number of sophisticated constructions which are species specific (Fig. 1);

- biosilica properties are closer rather to amorphous quartz than to silica gel from aqueous medium (Grachev et al., 2008);

- living organisms synthesize siliceous constructions at ambient conditions, without high temperatures and aggressive chemicals;

- understanding of molecular mechanisms of biosilicification gives not only valuable information of these organisms but also provides new knowledge in silicon and organic chemistry including approaches to new materials.

Siliceous particles and constructions are colorless and transparent which complicates their study with optical methods. These objects are electron dense and so are visible with transmittance electron microscopy but this method requires complex sampling and is not applicable for living specimens. Staining with vital fluorescent dyes is a powerful approach in biology which becomes more actual in last decades with dissemination of confocal microscopes. Rhodamines where the first dyes applied to staining growing cultures of diatoms (Li et al., 1989). These substances were accumulated in growing siliceous valves and girdle bands but the mature elements of siliceous frustules were colorless. The such behavior of rhodamines is explained with formation of silica in Silica Deposition Vesicles (SDVs) of diatoms. These intracellular organelles are close to lysosomes and their content is acidic $(\mathrm{pH}=5.5)$ (Vrieling et al., 1999) which stimulate accumulation of amines in these vesicles. Application of rhodamines allowed to obtain interesting data on diatom physiology (Safonova et al., 2007) and to synthesize new fluorescent materials by biotechnological approach (Kucki and Fuhrmann-Lieker, 2012). Unfortunately, rhodamines are not the convenient instrument because of low gap between staining and toxic concentrations. On the other hand, quantum yield of rhodamines in aqueous medium is high ( $>70 \%$ (Arbeloa et al., 1988) which results in high fluorescence from the cultivation medium.

A set of new dyes for vital staining of silicifying organisms was elaborated and this mini-review describes compounds based on 7-nitro-2,1,3-benzoxadiazole (NBD) fluorophore and more specific dyes which change fluorescent spectrum after incorporation into silica (PDMPO and coumarin derivatives).

*Corresponding author.

E-mail address: annenkov@lin.irk.ru (V.V. Annenkov)

(C) Author(s) 2018. This work is distributed under the Creative Commons Attribution 4.0 License. 

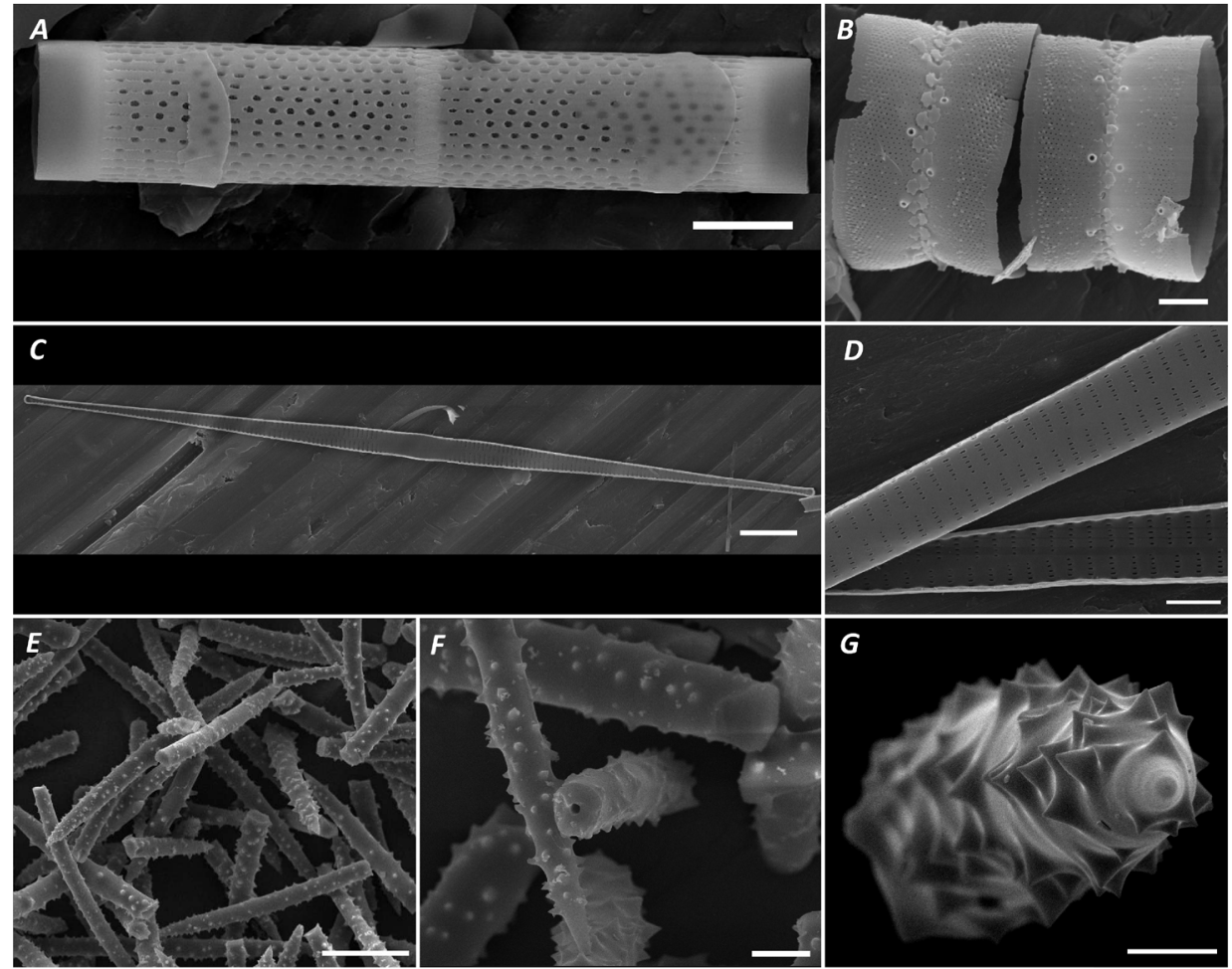

Fig. 1. Scanning electron microscopy images of diatom siliceous valves (A - Aulacoseira baikalensis (K. Meyer) Simonsen 1979; B - Stephanodiscus meyeri Genkal \& Popovskaya; C and D - Ulnaria ferefusiformis M. Kulikovskiy \& H. Lange-Bertalot); E-G - sponge spicules of Lubomirskia baicalensis (Pallas, 1773). Scale bar represents 50 (E), 10 (A, C and F), 5 (G) and 2 (B, D) $\mu$ m.

\section{NBD based dyes for vital staining}

The first NBD derivative for staining the growing siliceous frustules of diatoms was HCK-123 (Fig. 2) (Desclés et al., 2008). This dye contains a basic substituent which provides incorporation of the dye into acidic SDVs. NBD moiety is often applied for labeling of amine containing compounds (proteins, carbohydrates and etc.) with the use of 4-chloro-7-nitrobenzo-2-oxa1,3-diazole (Cl-NBD). This reagent is relatively cheap and readily reacts with primary and secondary amines.

Frustules of the diatom algae contain an interesting type of polyamines, so called long-chain polyamines (LCPAs). These compounds consist of amine groups separated by trimethylene fragments, with the first segment frequently containing four methylene groups (Kröger et al., 2000). Some amine groups are methylated, the LCPAs chains contain from several to
$>20$ nitrogen atoms. These substances are available in very limited amounts from the natural sources which complicates study of their properties and role in biosilicification. We have elaborated (Annenkov et al., 2006; 2009) a stepwise approach to synthesis of LCPAs, including individual compounds with 2-7 nitrogen atoms and oligomeric mixture containing polyamines with $>20$ nitrogen atoms.

The reaction of NBD-Cl with short polyamines (2-3 nitrogens) resulted in new dyes NBD-N2 and NBD-N3 (Fig. 2) (Annenkov et al., 2010). NBD-N3 as well as HCK-123 stains acidic organelles of eukaryotic cells but NBD-N2 is more selective and penetrates into growing siliceous structures only. This selectivity is explainable with shorter basic chain of NBD-N2 (one amine nitrogen only). NBD-N2 was successfully applied in study of initial stages of the diatom valve biosynthesis (Annenkov et al., 2013) and spiculogenesis<smiles>CN(C)CCNC(=O)CCCCCNc1ccc([N+](=O)[O-])c2nonc12</smiles><smiles>CN(C)CCCN(C)c1ccc([N+](=O)[O-])c2nonc12</smiles>

NBD-N2<smiles>CN(C)CCCN(C)CCCN(C)c1ccc([N+](=O)[O-])c2nonc12</smiles>

NBD-N3

Fig. 2. Structures of NBD based dyes: HCK-123, NBD-N2 and NBD-N3. 

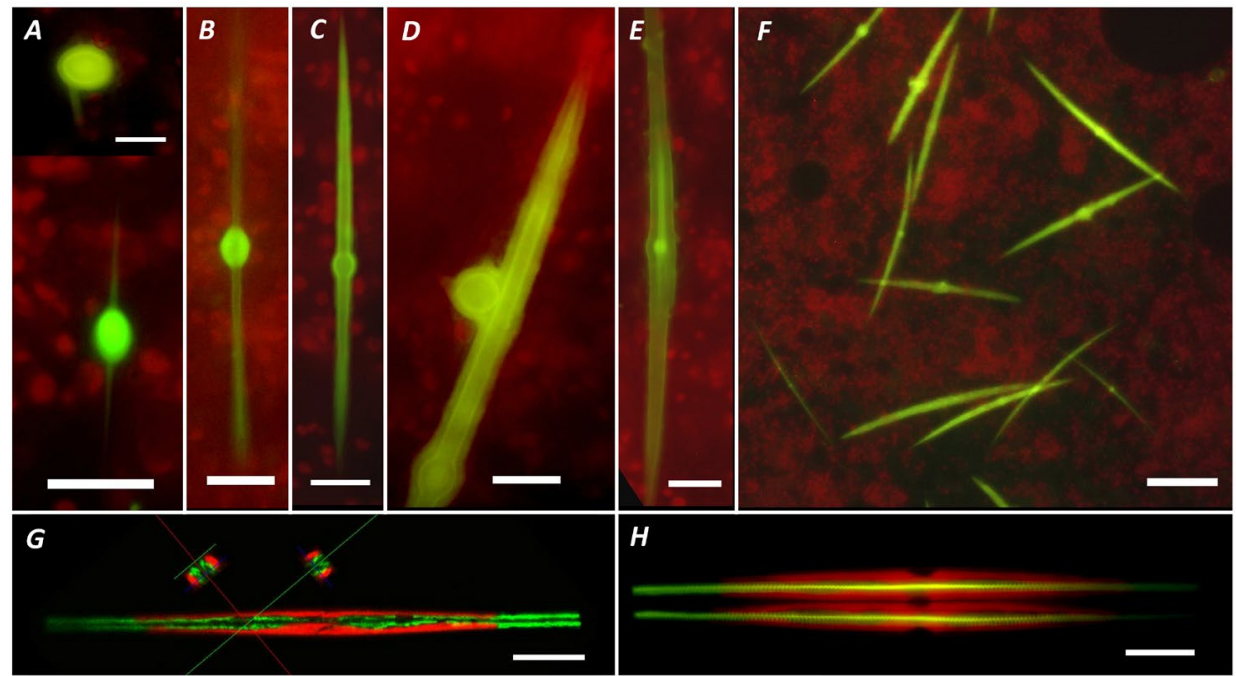

Fig. 3. Initial stages of sponge spicule (A-F, L. baicalensis) and diatom valve (G and H, U. ferefusiformis) growth visualized by cultivation in the presence on NBD-N2 dye. The main stages of spiculogenesis in L. baicalensis are: silicon accumulation in sclerocyte (A, top); formation of organic filament and protrusion of the new spicule from the cell (A, bottom and B); further elongation of the filament, sclerocyte capture silicic acid and organic substances from the extracellular space which allows further growth of the spicule (C); new sclerocytes merge with the growing spicule (D and E) and a mature spicules (F) are obtained. Submicrometer siliceous particles are formed in the diatom cytoplasm after 30-60 s after silicon addition to silicon free cultural medium (G) and after 10-15 min new siliceous valve is visible (H). Scale bar represents: 20 (F) and 10 (A-E, G and F) $\mu \mathrm{m}$.
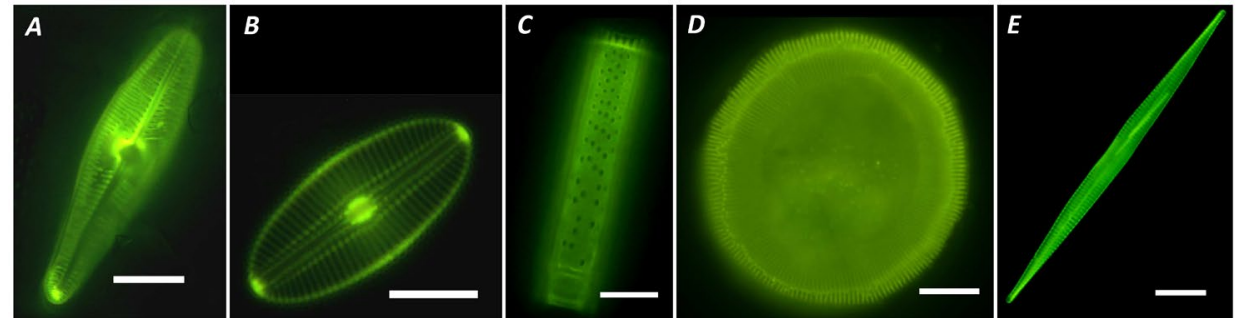

Fig. 4. Fluorescent images of diatom siliceous valves after cultivation in the presence of NBD-N2 dye. A-D - natural samples obtained from Lake Baikal (Russia), E - siliceous valve of $U$. ferefusiformis. Diatoms were cultivated for 3 days in the presence of $0.5 \mu \mathrm{M}$ NBD-N2 and the biomass was treated with $\mathrm{H}_{2} \mathrm{SO}_{4} / \mathrm{H}_{2} \mathrm{O}_{2}(1: 1)$ mixture. Scale bar represents $10 \mu \mathrm{m}$.

in sponge L. baicalensis (Annenkov and Danilovtseva, 2016). Fluorescent and confocal microscopy allowed to visualize primary submicrometer silicon-containing particles formed during capture of silicic acid with diatoms and growing spicules starting from single sponge cell (Fig. 3).

NBD-N2 and NBD-N3 penetrates into siliceous frustules of diatoms and sponge spicules (Fig. 4-6). The dyes are deeply buried in the biogenic silica and retain fluorescent activity after treatment with strong acids and oxidizing agents during cleaning of organic substances. The stained valves and spicules are suitable for study with confocal microscopy (Fig. 6) which allows to obtain additional information about internal structure of the material without real slicing.

Fig. 5. Fluorescent images of diatom siliceous valves and sponge spicules after cultivation in the presence of NBD-N2 dye. A - L. baicalensis, B - Baikalospongia bacillifera (Dybowsky, 1880), C - Swartschewskia papyracea (Dybowsky, 1880) and D - Baikalospongia intermedia (Dybowsky, 1880). Sponges were cultivated for a month in the presence of $0.5 \mu \mathrm{M}$ NBD-N2 and the biomass was treated with bleach. Fluorescence allows to distinguish spicules grown during the experiment from old spicules. Scale bar represents $50 \mu \mathrm{m}$.

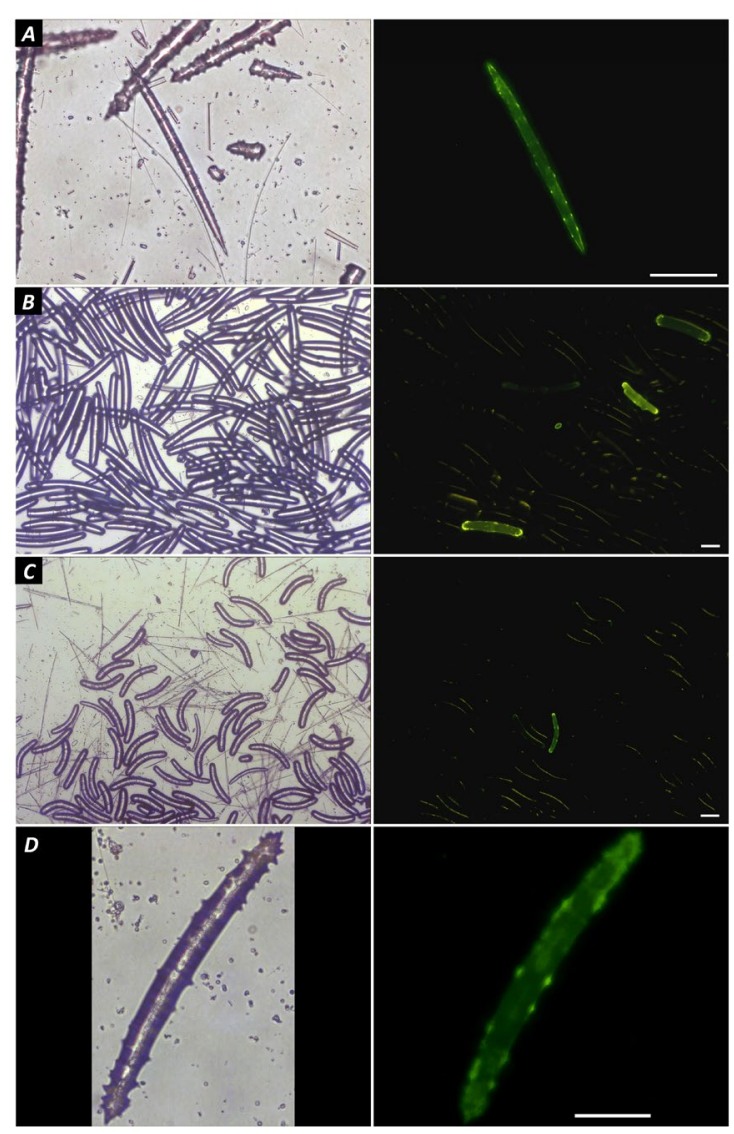




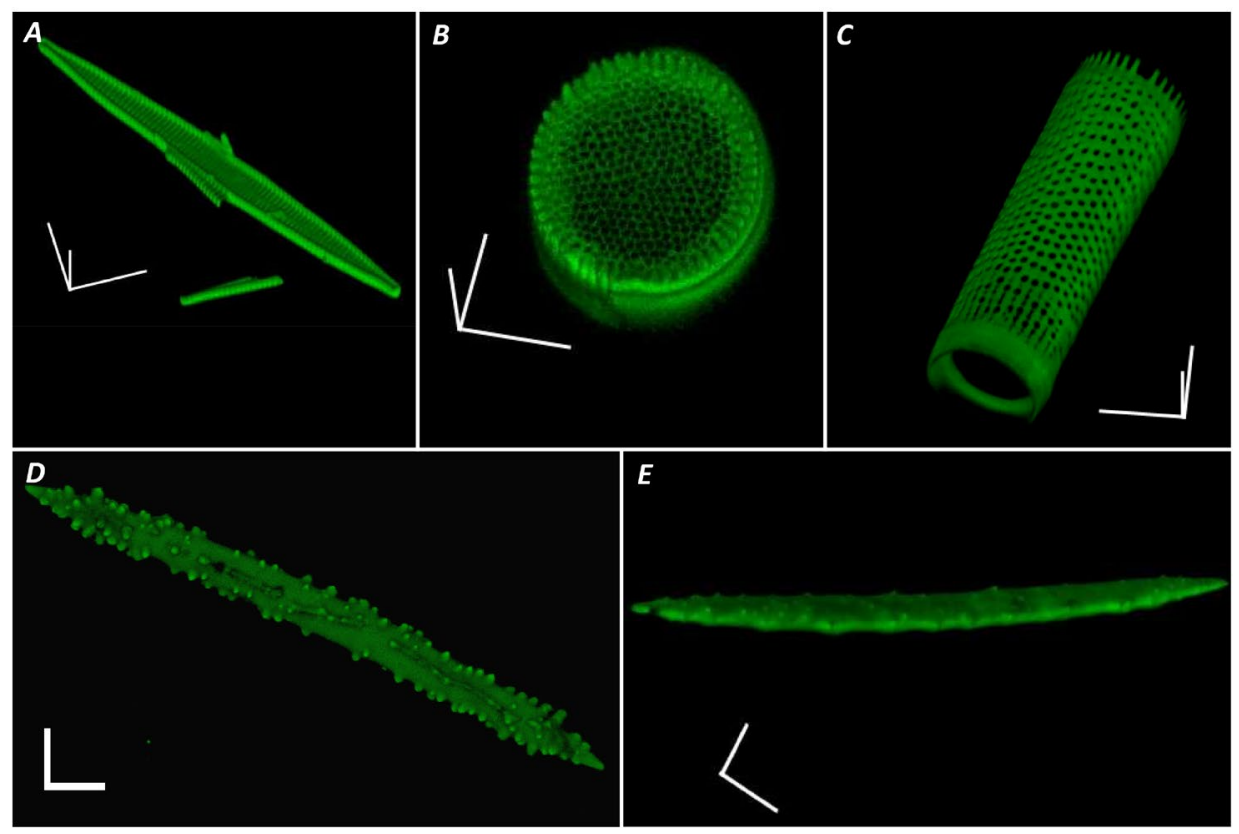

Fig. 6. Confocal microscopy 3D images of diatom siliceous valves and sponge spicules after cultivation in the presence of NBD-N2 dye. A - siliceous valve of $U$. ferefusiformis, B and C - siliceous valve of diatom Aulacoseira sp., D and E - siliceous spicules of $L$. baicalensis sponge. Scale bar represents 10 (A-C) and 20 (D and E) $\mu \mathrm{m}$.

The vital fluorescent staining is especially valuable in the case of sponges. These multicellular invertebrates have a skeleton from needle-like spicules which are fastened with organic compounds. Sponge cells and a lot of symbionts live inside this skeleton. The spicule shape and peculiarities of the spicule growth (spiculogenesis) are important for the sponge classification and as a marker of sponge growth and health in various experiments. Our works (Annenkov et al., 2014; 2016) give new powerful tool for study of the siliceous sponges. The siliceous spicules are colorless and transparent which decrease efficiency of light microscopy in their study but fluorescent spicules are a good object for confocal microscopy (Fig. 6). Staining with NBD-N2 dye allowed us to see growing spicules from the single silicon-enriched cells (Fig. 3) (Annenkov et al., 2016). This study was done with the whole sponges or sponge cultures (primmorphs) without isolation of the growing spicules because the fluorescent staining allows to observe these objects surrounded by other cells and mature spicules.

Study of the sponge physiology and influence of various ecological conditions on the sponges requires methods of the spiculogenisis control. This is not an ordinary task because any sponge and primmorph contain old spicules and how to distinguish between old spicules and new ones, formed during experiment? The vital staining with the fluorescent nontoxic dye is the easy and accurate method to do this (Fig. 5).

\section{Fluorescent derivatives of long chain polyamines}

Study of NBD-Cl interaction with LCPAs (more than 4 nitrogen atoms) resulted in unexpected reaction of NBD-Cl with tertiary nitrogen atoms giving rise to NBD derivatives and unsaturated compounds (Annenkov et al., 2015). A set of new dyes containing relatively long polyamine substituents ( $\geq 3$ nitrogen atoms) was prepared in this work (Fig. 7, Annenkov et al., 2018). The longer polyamine chain in these dyes increase activity in association with silica due to coordination with surface silanol groups. This property of the new dyes is useful in fluorescent staining of diatom frustules, siliceous spicules and silica containing natural sediments (Fig. 8). In the latter case the siliceous particles become easy distinguishable from non-stained terrigeneous admixtures.

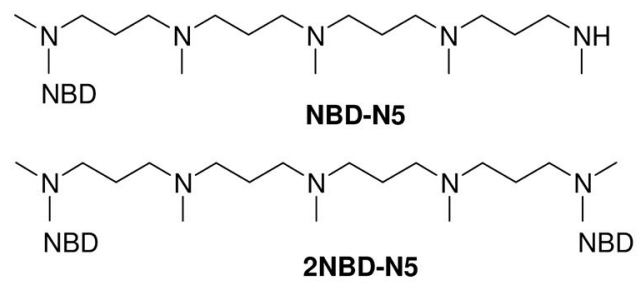

2NBD-N4

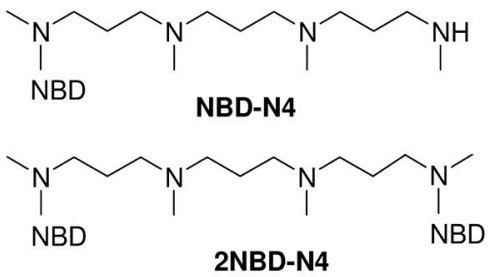<smiles>CNCCC[N+](C)(C)CCC[N+](C)(C)C</smiles><smiles>CN(CCC[N+](C)(C)CCC[N+](C)(C)N(C)C)[NH+](C)C</smiles>
NBD $n=4-22$<smiles>Cc1ccc([N+](=O)[O-])c2nonc12</smiles>

Fig. 7. Structures of fluorescent dyes based on long chain polyamines. 

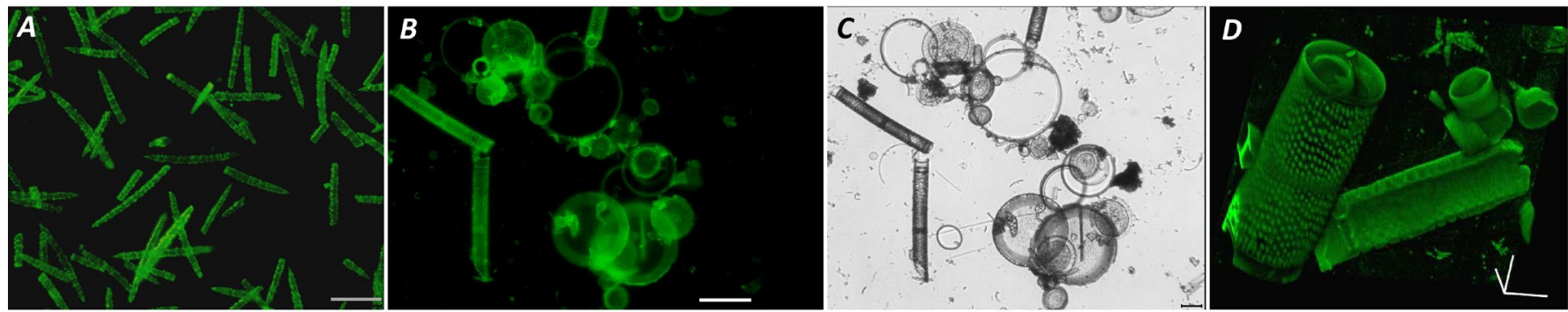

Fig. 8. Siliceous materials stained with fluorescent dyes based on long chain polyamines. A - L. baicalensis spicules, B-D Baikalean sediment. Scale bar represents 100 (A), 20 (B and C) and 10 (D) $\mu \mathrm{m}$.<smiles>CCN(CC)c1ccc2cc(C(=O)N(C)CCCN(C)C)c(=O)oc2c1</smiles><smiles>CN(C)CCNC(=O)COc1ccc(-c2cnc(-c3ccncc3)o2)cc1</smiles>

PDMPO

Fig. 9. Structure of Q-N2 and PDMPO dyes.

\section{Dyes with variable fluorescent properties}

As discussed above, NBD based dyes stain acidic cell vesicles and SDV due to specific structure of the substituent at fluorophore group. The next generation of fluorescent dyes for vital staining of siliceous structures in living cells changes fluorescent spectrum under association with silica. The first representative of these dyes is 2-(4-pyridyl)-5-((4-(2-dimethylaminoethylaminocarbamoyl)methoxy)-phenyl)oxazole (PDMPO, Fig. 9) (Shimizu et al., 2001; Parambath et al., 2016). Fluorescence of PDMPO depends on $\mathrm{pH}$ and the emission spectrum changes under association with silanol groups or involving into acidic vesicles. This behavior is useful in study of silicifying organisms as well as in study of silicification reactions in vitro.

Recently we have synthesized a new coumarin based fluorescent dye Q-N2 (Fig. 9) which contain amine group in the substituent (Annenkov et al., 2019). In contrast to PDMPO, fluorescence spectrum of this dye in aqueous medium does not depend on $\mathrm{pH}$ value. Aggregation of Q-N2 with silica or entrapping into siliceous materials results in enhancing of blue fluorescence and appearance of green emission. These effects were studied with fluorescence microscopy and they are easy visible with a routine epifluorescence microscope: fluorescence color changes from blue to cyan under excitation at $350-360 \mathrm{~nm}$ and green fluorescence appears under excitation at $470 \mathrm{~nm}$ (Fig. 10).

Fig. 10. Change of Q-N2 emission spectrum in the presence of $100 \mathrm{mM}$ silicic acid during condensation at $\mathrm{pH} 7$ (top spectrum - excitation at $365 \mathrm{~nm}$, bottom spectrum - excitation at $470 \mathrm{~nm}$ ), the data show faster growth of the fluorescence intensity in green area. The microscopy images show cells of Aulacoseira sp. after cultivation in the presence of 0.5 $\mu \mathrm{M}$ Q-N2, top - light image, middle - excitation at $370 \mathrm{~nm}$, bottom - excitation at $470 \mathrm{~nm}$. Scale bar represents $10 \mu \mathrm{m}$.
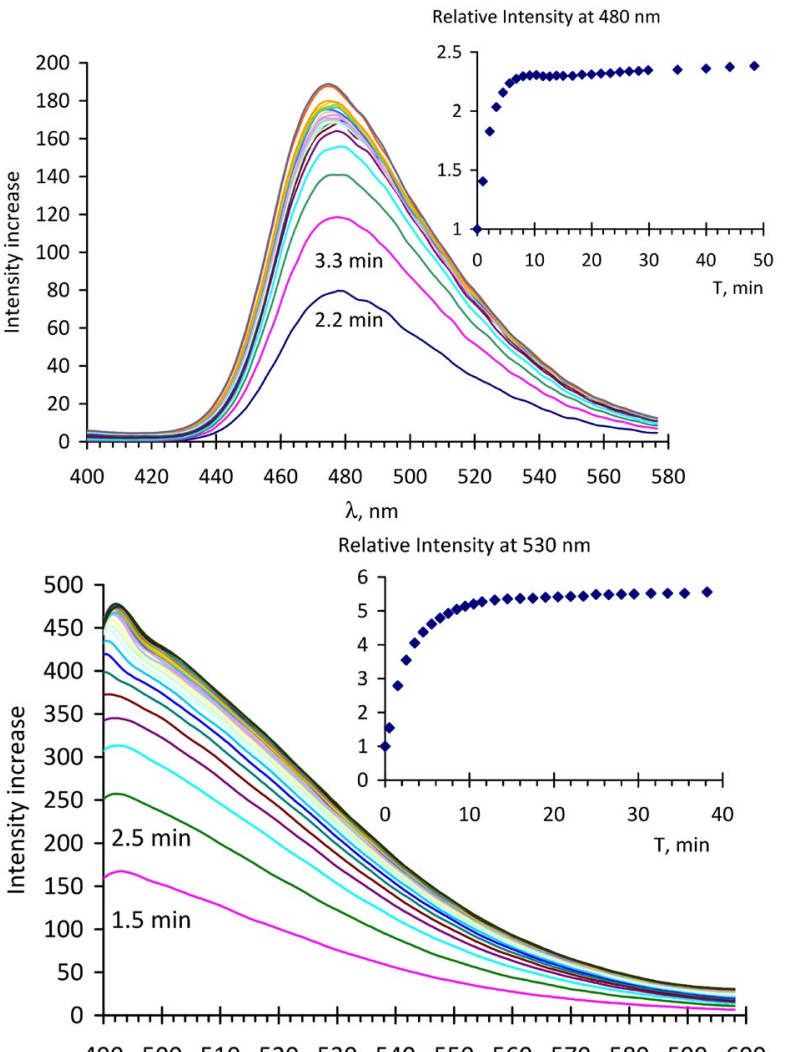

$\begin{array}{llllllllllll}490 & 500 & 510 & 520 & 530 & 540 & 550 & 560 & 570 & 580 & 590 & 600\end{array}$

$\lambda, \mathrm{nm}$

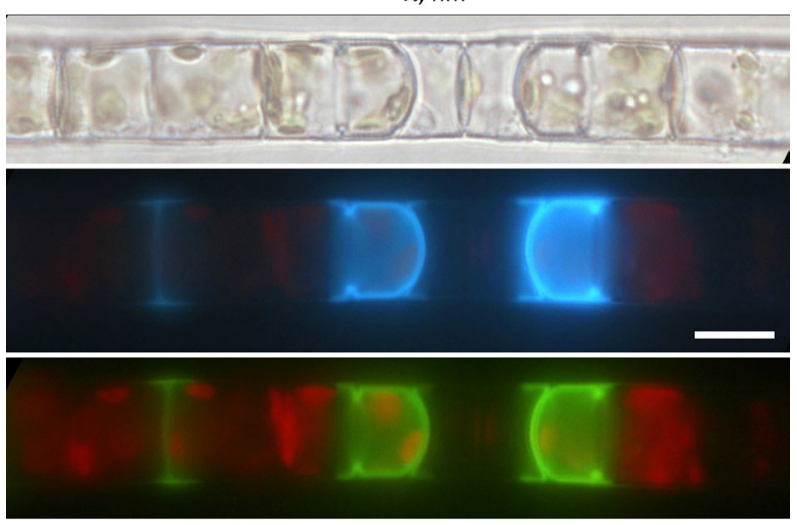


The absence of $\mathrm{pH}$-depending changes of the Q-N2 fluorescence allows to attribute the strong cyan (green) fluorescence to siliceous structures. The other advantage of Q-N2 is the excitation maximum below $400 \mathrm{~nm}$, far from excitation of chloroplasts. In combination with high quantum yield (11\% comparing with $<1 \%$ for NBD derivatives) this decreases damaging effect on living cells during microscopy investigation. Q-N2 shows inhibitory effect on the diatom growth in $10 \mu \mathrm{M}$ concentration (20-30\% inhibition) which is 20 times higher its staining concentration. Q-N2 stains growing siliceous structures in diatoms and sponges (Fig. 11) and video monitoring of the silicon capture allowed us to find formation of condensed oligosilicates in several seconds after silicon capture with the diatom cell (Annenkov et al., 2019). This observation confirm hypothesis about silicon assimilation by diatoms in the form of partially condensed silicic acid.

\section{Acknowledgments}

This work was supported by the Russian Academy of Sciences (project \# AAAA-A16-116122110059-3); E. Danilovtseva thanks the Russian Foundation for Basic Research (Project \# 16-04-00799 a). The authors wish to thank the Center of Ultramicroanalysis (Limnological Institute), the Baikal Analytical Center (Irkutsk Institute of Chemistry) and the Isotope-geochemical research center for Collective Use (A. P. Vinogradov Institute of Geochemistry of the Siberian Branch of the Russian Academy of Sciences) for providing equipment. We are deeply appreciative of I. Khanaev for the sponge samples and V. Itskovich for identification of the sponge species.

\section{References}

Annenkov V.V., Patwardhan S.V., Belton D. et al. 2006. A new stepwise synthesis of a family of propylamines derived from diatom silaffins and their activity in silicification. Chemical Communications 14: 1521-1523. DOI: 10.1039/ b515967a

Annenkov V.V., Zelinskiy S.N., Danilovtseva E.N. et al. 2009. Synthesis of biomimetic polyamines. ARKIVOC xiii: 116-130. DOI: 10.3998/ark.5550190.0010.d10

Annenkov V.V., Danilovtseva E.N., Zelinskiy S.N. et al. 2010. Novel fluorescent dyes based on oligopropylamines for the in vivo staining of eukaryotic unicellular algae. Analytical Biochemistry 407: 44-51. DOI: 10.1016/j.ab.2010.07.032

Annenkov V.V., Basharina T.N., Danilovtseva E.N. et al. 2013. Putative silicon transport vesicles in the cytoplasm of the diatom Synedra acus during surge uptake of silicon. Protoplasma 250: 1147-1155. DOI 10.1007s00709-013-0495-x

Annenkov V.V., Glyzina O.Yu., Verkhozina O.N. et al. 2014. Fluorescent amines as a new tool for study of siliceous sponges. Silicon 6: 227-231. DOI 10.1007/s12633-0149220-4

Annenkov V.V., Verkhozina O.N., Shishlyannikova T.A. et al. 2015. Application of 4-chloro-7-nitrobenzo-2-oxa-1,3diazole in analysis: fluorescent dyes and unexpected reaction with tertiary amines. Analytical Biochemistry 486: 5-13. DOI: $10.1016 /$ j.ab.2015.06.025
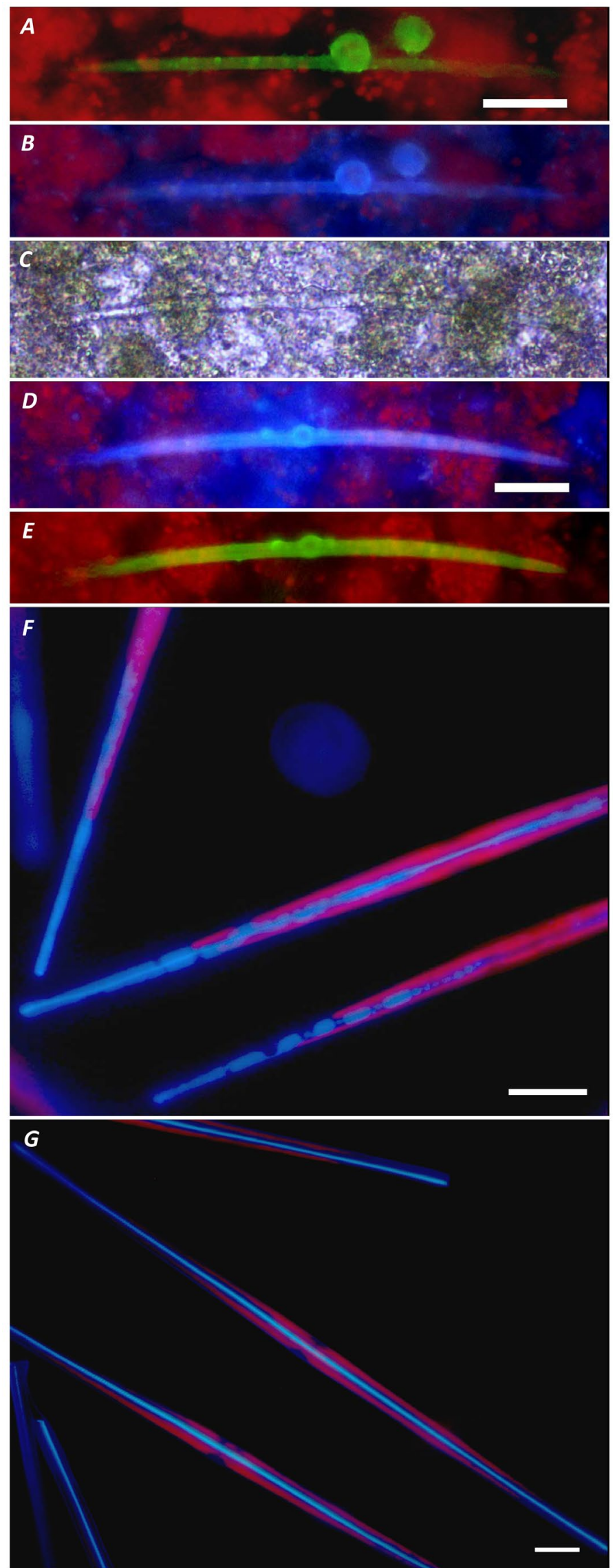

Fig. 11. Initial stages of sponge spicules (A-E, L. baicalensis) and diatom valve (F and G, U. ferefusiformis) growth monitored with Q-N2 dye. The cyan color of new siliceous structures is distinguishable from blue fluorescence of free Q-N2. Scale bar represents 20 (A-E) and 10 (F and G) $\mu \mathrm{m}$. 
Annenkov V.V., Danilovtseva E.N. 2016. Spiculogenesis in the siliceous sponge Lubomirskia Baicalensis studied with fluorescent staining. Journal of Structural Biology 194: 2937. DOI:10.1016/j.jsb.2016.01.010

Annenkov V., Danilovtseva E., Pal'shin V. et al. 2018. Fluorescently-tagged polyamines for the staining of siliceous materials. Plant Physiology and Biochemistry 125: 205-211. DOI: 10.1016/j.plaphy.2018.02.014

Annenkov V.V., Zelinskiy S.N., Pal'shin V.A. et al. 2019. Coumarin based fluorescent dye for monitoring of siliceous structures in living organisms. Dyes and Pigments 160: 336343. DOI:10.1016/j.dyepig.2018.08.020

Arbeloa F.L., Ojeda P.R., Arbeloa I.L. 1988. Dimerization and trimerization of rhodamine $6 \mathrm{G}$ in aqueous solution. Journal of the Chemical Society-Faraday Transactions II 84: 1903-1912. DOI: 10.1039/F29888401903

Desclés J., Vartanian M., El Harrak A. et al. 2008. New tools for labeling silica in living diatoms. New Phytologist 177: 822-829. DOI: 10.1111/j.1469-8137.2007.02303.x

Grachev M.A., Annenkov V.V., Likhoshway Y.V. 2008. Silicon nanotechnologies of pigmented heterokonts. BioEssays 30: 328-337. DOI: 10.1002/bies.20731

Kucki M., Fuhrmann-Lieker T. 2012. Staining diatoms with rhodamines dyes: control of emission colour in photonic biocomposites. Journal of the Royal Society Interface 9: 727-733. DOI: $10.1098 /$ rsif.2011.0424

Kröger N., Deutzmann R., Bergsdorf C. et al. 2000. Spe- cies-specific polyamines from diatoms control silica morphology. Proceedings of the National Academy of Sciences of the United States of America 97: 14133-14138. DOI: 10.1073/ pnas. 260496497

Li C.-W., Chu S., Lee M. 1989. Characterizing the silica deposition vesicle of diatoms. Protoplasma 151: 158-163. DOI: $10.1007 / \mathrm{BF} 01403453$

Parambath M., Hanley Q.S., Martin-Martinez F.J. et al. 2016. The nature of the silicaphilic fluorescence of PDMPO. Physical Chemistry Chemical Physics 18: 5938-5948. DOI: 10.1039/C5CP05105C

Treguer P., Nelson D.M., Van Bennekom A.J. et al. 1995. The silica balance in the world ocean: a reestimate. Science 268: 375-379. DOI: 10.1126/science.268.5209.375

Vrieling E.G., Gieskes W.W.C., Beelen T.P.M. 1999. Silicon deposition in diatoms: control by the $\mathrm{pH}$ inside the silicon deposition vesicle. Journal of Phycology 35: 548-559. DOI: 10.1046/j.1529-8817.1999.3530548

Safonova T.A., Annenkov V.V., Chebykin E.P. et al. 2007. Aberration of morphogenesis of siliceous frustule elements of the diatom Synedra acus in the presence of germanic acid. Biochemistry (Moscow) 72: 1261-1270. DOI: 10.1134/ S0006297907110132

Shimizu K., Del Amo Y., Brzezinski M.A. et al. 2001. A novel fuorescent silica tracer for biological silicifcation studies. Chemistry \& Biology 8: 1051-1060. DOI: 10.1016/S10745521(01)00072-2 